\title{
Realization of the Linear Tree that Corresponds to a Fundamental Loop Matrix
}

\author{
Jianping QIAN ${ }^{1}$, Peng-Yung WOO ${ }^{2}$ \\ ${ }^{1}$ Department of Automation, Nanjing University of Science and Technology, Nanjing, China \\ ${ }^{2}$ Department of Electrical Engineering, Northern Illinois University, Dekalb, USA \\ Email:pwoo@niu.edu
}

Received October 13, 2009; revised October 27, 2009; accepted October 28, 2009

\begin{abstract}
Graph realization from a matrix is an important topic in network topology. This paper presents an algorithm for the realization of a linear tree based on the study of the properties of the number of the single-link loops that are incident to each tree branch in the fundamental loop matrix $\mathbf{B}_{\mathbf{f}}$. The proposed method judges the pendent properties of the tree branches, determines their order one by one and then achieves the realization of the linear tree. The graph that corresponds to $\mathbf{B}_{\mathbf{f}}$ is eventually constructed by adding links to the obtained linear tree. The proposed method can be extended for the realization of a general tree.
\end{abstract}

Keywords: Fundamental Loop Matrix, Linear Tree, Graph

\section{Introduction}

Graph realization from a matrix is an important topic in network topology. It has a broad application in electrical networks, switching networks, linear programming etc. The study of graph realization from a matrix is to judge whether a given matrix can be realized to be a graph. If yes, the method for realization needs to be found so that graph realization for a given matrix can be achieved. There are three aspects in graph realization from a matrix: a) the realizablity of the given matrix, b) the method for graph realization from a matrix, c) the unique correspondence between the realized graph and the given matrix. All the above issues have been under research by using algebra theory, graph theory, geometry structure etc [1-5]. While the necessary and sufficient conditions for the realizability of a graph from a matrix are proposed by many researchers from different viewpoints, the judgment of the realizability and the realization of the graph, in practice, are carried out simultaneously rather than in sequence.

Quite a few researchers studied the realization of a linear tree [6,7]. This paper presents an algorithm for the realization of a linear tree based on the study of the properties of the number of the single-link loops that are incident to each tree branch in the fundamental loop matrix $\mathbf{B}_{\mathbf{f}}$. The proposed method judges the pendent properties of the tree branches, determines their order one by one and then achieves the realization of the linear tree.
Since a general tree possesses a linear sub-tree, a general tree can then be realized by adding other tree branches after the linear sub-tree is realized. The graph that corresponds to an arbitrary fundamental loop matrix $\mathbf{B}_{\mathbf{f}}$ is eventually constructed by adding links to the obtained linear tree. As is seen, the proposed method is simple, practical and efficient in realizing a general tree.

\section{Pretreatment}

For a graph that possesses $n$ nodes and $b$ branches, after a certain tree $T$ is chosen, the fundamental loop matrix $\mathbf{B}_{\mathbf{f}}$ has a standard form $\left[\mathbf{B}_{\mathbf{t}} \mathbf{1}\right]$ where $\mathbf{B}_{\mathbf{t}}$ is a $(b-n+1) \times(n-1)$ matrix. Assume that the columns $b_{1}, b_{2}, \ldots, b_{n-1}$ in $\mathbf{B}_{\mathrm{t}}$ correspond to the branches $t_{1}, t_{2}, \ldots, t_{n-1}$ of $T$, while the columns $b_{n}, b_{n+1}, \ldots, b_{b}$ in $\mathbf{1}$ correspond to the links. As is known, $b_{i}^{\mathrm{T}} \cdot b_{j}$ indicates the number of the pairs of corresponding entries being all " 1 "s in the branch columns $b_{i}$ and $b_{j}$. By realizing a graph from the matrix that is constructed by the rows of the aforementioned pairs as well as the same indexed rows in the corresponding link columns, we know that $b_{i}^{\mathrm{T}} \cdot b_{j}$ is the number of the single-link loops that pass $t_{i}$ and $t_{j}$. Specially, $b_{i}^{\mathrm{T}} \cdot b_{i}$ indicates the number of entries " 1 " in $b_{i}$. By realizing a graph from the matrix that is constructed by the rows of the aforementioned "1"s as well as the same indexed rows in the corresponding link columns, we know that $b_{i}^{\mathrm{T}} \cdot b_{i}$ is the number of the single-link loops that pass $t_{i}$. To facilitate 
our discussions, it is assumed that the tree that corresponds to $\mathbf{B}_{\mathbf{t}}$ is a linear tree. For example:

$$
\begin{aligned}
& \begin{array}{llllll}
1 & 2 & 3 & 4 & 5 & 6
\end{array}
\end{aligned}
$$

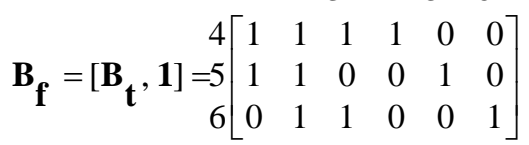

The number of the pairs of corresponding entries being all " 1 "s in $b_{1}$ and $b_{2}$ (i.e., row 1 and row 2 ) is 2 . Thus, $b_{1}^{\mathrm{T}} \cdot b_{2}=2$. By realizing a graph (Figure 1 ) from the matrix

$$
\begin{array}{llll}
1 & 2 & 4 & 5
\end{array}
$$

$4\left[\begin{array}{llll}1 & 1 & 1 & 0\end{array}\right]$ that is constructed by the rows of the $5\left[\begin{array}{llll}1 & 1 & 0 & 1\end{array}\right]$

aforementioned pairs as well as the same indexed rows in the corresponding link columns, we know that $b_{1}{ }^{\mathrm{T}} \cdot b_{2}=2$ is the number of the single-link loops that pass $t_{1}$ and $t_{2}$. Specially, $b_{1}{ }^{\mathrm{T}} \cdot b_{1}=2$ is the number of the single-link loops that pass $t_{1}$. The following theorems present the relationship among the branches of $T$ (Due to limitation of space, the proofs are not presented in this article).

Theorem 1: Suppose $\mathbf{B}_{\mathbf{t}}$ is a $(b-n+1) \times 3$ matrix and $t_{p}$ is a pendent branch of the linear tree $T$ that corresponds to

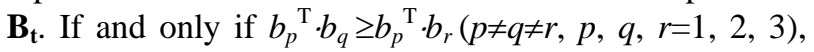
the order of the branches of $T$ is $t_{p}, t_{q}, t_{r}$.

As is seen in Figure 2, while there is only one single-link loop passing $t_{p}$ and $t_{q}$, there are two passing $t_{p}$ and $t_{r}$. Therefore, $b_{p}{ }^{\mathrm{T}} \cdot b_{r} \geq b_{p}{ }^{\mathrm{T}} \cdot b_{q}$ and the order of the branches of $T$ is $t_{p}, t_{r}, t_{q}$ according to Theorem 1 .

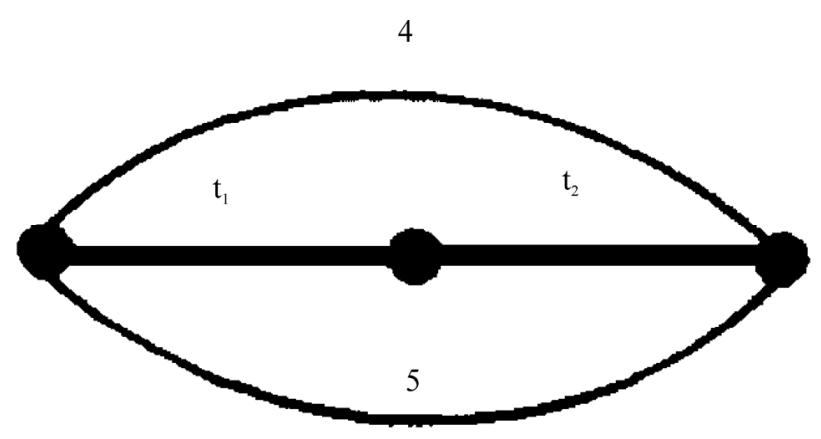

Figure 1. The explanation of $\boldsymbol{b} \cdot \boldsymbol{b}_{\mathrm{j}}$.

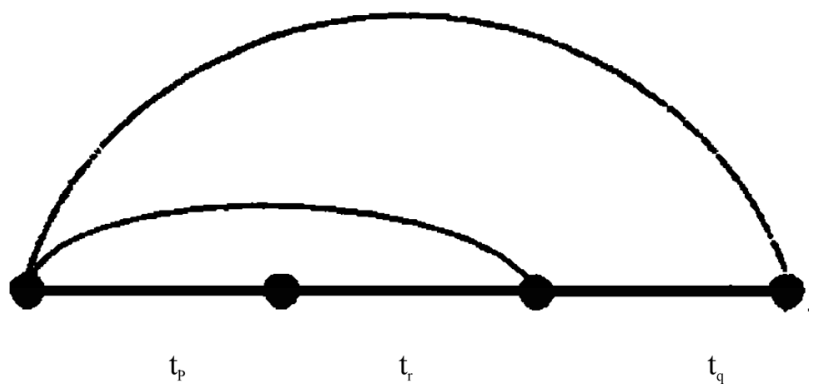

Figure 2. The explanation of Theorem 1.
Theorem 2: Suppose $\mathbf{B}_{\mathbf{t}}$ is a $(b-n+1) \times(n-1)$ matrix and $t_{p}$ is a pendent branch of the linear tree $T$ that corresponds to $\mathbf{B}_{\mathbf{t}}$. If and only if $b_{p}{ }^{\mathrm{T}} \cdot b_{q} \geq b_{p}{ }^{\mathrm{T}} \cdot b_{r}(p \neq q \neq r, 1 \leq p, q$, $r \leq n-1)$, the order of the branches of $T$ is $t_{p}, \ldots, t_{q}, \ldots, t_{r}$ (i.e., $t_{q}$ is closer to $t_{p}$ than $t_{r}$ is).

Theorem 3: Suppose $\mathbf{B}_{\mathbf{t}}$ is a $(b-n+1) \times(n-1)$ matrix. For a certain column $b_{l}$ arbitrarily chosen, if $b_{l}^{\mathrm{T}} \cdot b_{p}$ $=\min _{i \neq l, i=1,2, \ldots n-1} b_{l}^{\mathrm{T}} \cdot b_{i}(p \neq l, 1 \leq p \leq n-1), t_{p}$ is a pendent branch of the linear tree $T$ that corresponds to $\mathbf{B}_{\mathbf{t}}$.

Theorem 4: Suppose $\mathbf{B}_{\mathbf{t}}$ is a $(b-n+1) \times(n-1)$ matrix. For a certain column $b_{l}$ arbitrarily chosen, if $b_{l}^{\mathrm{T}} \cdot b_{j}$ $=\min _{i \neq l, i=1,2, \ldots n-1} b_{l}^{\mathrm{T}} \cdot b_{i}$ where $j \in U=\{p, q, r, \ldots, w\}$ $(p \neq q \neq r \neq \ldots \neq w \neq l, 1 \leq p, q, r, \ldots, w \leq n-1)$, there must exist a certain $s \in U$ such that $t_{\mathrm{s}}$ is a pendent branch of the linear tree $T$ that corresponds to $\mathbf{B}_{\mathbf{t}}$.

As is seen in Figure 3 , while $b_{l}^{\mathrm{T}} \cdot b_{m}=b_{l}^{\mathrm{T}} \cdot b_{n}=2, b_{l}^{\mathrm{T}} \cdot b_{p}$ $=b_{l}^{\mathrm{T}} \cdot b_{q}=1$. Thus, there must exist a certain $s \in U=\{p, q\}$ such that $t_{p}$ or $t_{q}$ is a pendent branch of the linear tree $T$.

Theorem 5: Suppose $\mathbf{B}_{\mathbf{t}}$ is a $(b-n+1) \times(n-1)$ matrix. For a certain column $b_{l}$ arbitrarily chosen, if $b_{l}^{\mathrm{T}} \cdot b_{j}$ $=\min _{i \neq l, i=1,2, \ldots n-1} b_{l}^{\mathrm{T}} \cdot b_{i}$ where $j \in U=\{p, q, r, \ldots, w\}$ $(p \neq q \neq r \neq \ldots \neq w \neq l, 1 \leq p, q, r, \ldots, w \leq n-1)$, then the $t_{h}, t_{m}$, $t_{n}, \ldots, t_{l}$ that correspond to $\bar{U}=\{h, m, n, \ldots, l\}$ construct a linear sub-tree of $T$, where $U \cap \bar{U}=\phi$ and $\mid U$ $\cup \bar{U} \mid=n-1$.

As is seen in Figure 3, $U=\{p, q\}$ and $\bar{U}=\{m, n, l\}$. Since $b_{l}^{\mathrm{T}} \cdot b_{m}=b_{l}^{\mathrm{T}} \cdot b_{n}=2$ but $b_{l}^{\mathrm{T}} \cdot b_{p}=b_{l}^{\mathrm{T}} \cdot b_{q}=1, t_{m}, t_{n}$ and $t_{l}$ construct a linear sub-tree of $T$.

Theorem 6: Suppose $\mathbf{B}_{\mathbf{t}}$ is a $(b-n+1) \times(n-1)$ matrix. For a certain column $b_{l}$ arbitrarily chosen, $b_{l}^{\mathrm{T}} \cdot b_{j}$ $=\min _{i \neq l, i=1,2, \ldots n-1} b_{l}^{\mathrm{T}} \cdot b_{i}$ where $j \in U=\{p, q, r, \ldots, w\}$ $(p \neq q \neq r \neq \ldots \neq w \neq l, 1 \leq p, q, r, \ldots, w \leq n-1)$. Thus, the $t_{h}, t_{m}$, $t_{n}, \ldots, t_{l}$ that correspond to $\bar{U}=\{h, m, n, \ldots, l\}$ construct

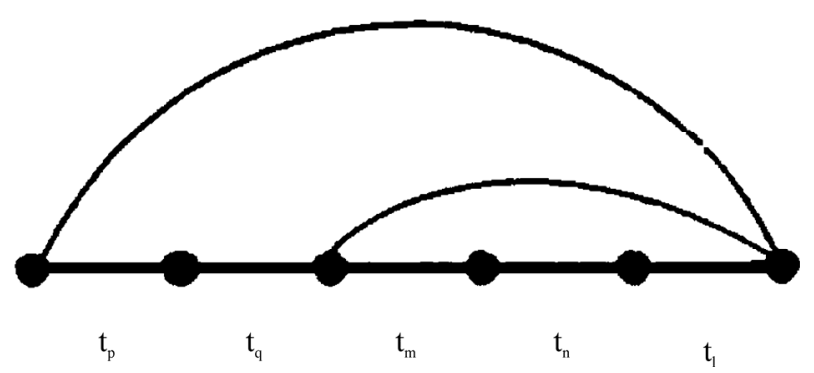

Figure 3. The explanation of Theorem 4. 
a linear sub-tree of $T$, where $U \cap \bar{U}=\phi$ and $\mid U$ $\cup \bar{U} \mid=n-1$. Then for a certain $k \in \bar{U}$, if there are certain $s_{1}$ and $s_{2} \in U^{*} \subseteq U$ such that $b_{k}{ }^{T} \cdot b_{s_{1}}=b_{k}{ }^{T} \cdot b_{s_{2}}$, after the links $L$ 's that correspond to $\max _{k=h, m, n, \ldots, l} b_{k}{ }^{T} \cdot b_{s_{1}}$ in the graph $\mathrm{G}$ that corresponds to $\mathbf{B}_{\mathbf{f}}$ are deleted, the graph constructed by $G_{1}$ that corresponds to $U^{*}$ and the graph $\mathrm{G}_{2}$ that corresponds to $\left(U-U^{*}\right) \cup \bar{U}$ is a separable graph, where $G_{1}$ and $G_{2} \subset G, G_{1} \cup G_{2} \cup G_{L}=G, G_{1} \cap G_{2} \cap G_{L}=\phi$ and $G_{L}$ is the graph that corresponds to the links $L$ 's. If $G_{1}$ and $G_{2}$ are inseparable graphs, respectively, then $G_{1}$ and $\mathrm{G}_{2}$ have a common cut-point.

As is seen in Figure 4(a), $b_{l}^{\mathrm{T}} \cdot b_{j}=1$ where $j \in U=\{p, q, r$, $w\}$. Thus, the $t_{h}, t_{m}, t_{n}, \ldots, t_{l}$ that correspond to $\bar{U}=\{h, m$, $n, l\}$ construct a linear sub-tree of $T$. Let $k=h \in \bar{U}$, and $s_{1}$ and $s_{2} \in U^{*}=\{p, q\} \subseteq U$. Since $b_{k}{ }^{T} \cdot b_{s_{1}}=b_{k}{ }^{T} \cdot b_{s_{2}}=3$, links 1,2 and 3 are deleted. The remaining $G_{1}$ and $G_{2}$ are separable graphs as shown in Figure 4(b).

From the above facts, it is seen that when the conditions in Theorem 6 are satisfied, $\mathrm{G}_{1}$ and $\mathrm{G}_{2}$ are 2-isomorphic. Therefore, in the ordering of the branches of the linear tree, the branches of the linear sub-tree that corresponds to $G_{1}$ are first put into order separately, then those of the linear sub-tree that corresponds to $G_{2}$. The ordering of the branches of the linear tree is now reduced to the ordering of the branches for each linear sub-tree. The solution of this problem is depending on the following Theorem 7, where it is assumed that the sub-trees do not form 2-isomorphism.

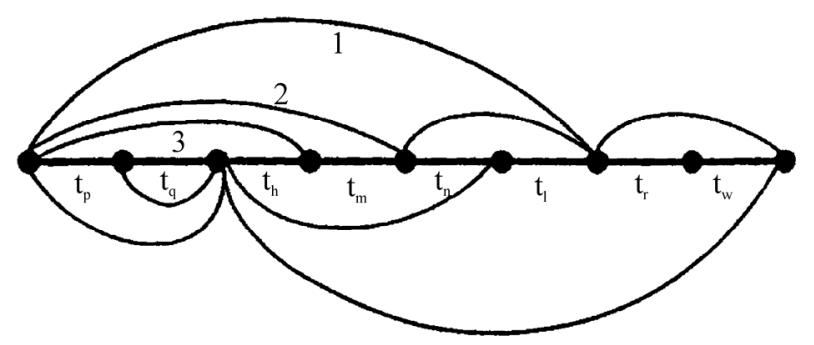

(a)

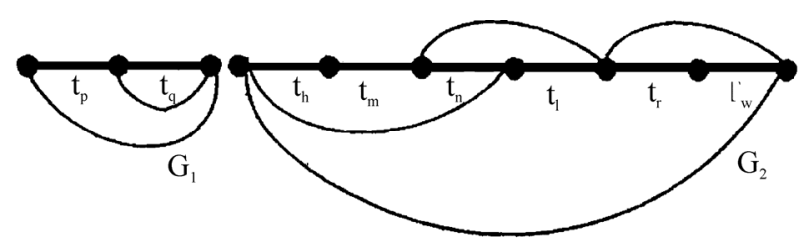

(b)

Figure 4. The explanation of Theorem 6.
Theorem 7: Suppose $\mathbf{B}_{\mathbf{t}}$ is a $(b-n+1) \times(n-1)$ matrix. For a certain column $b_{l}$ arbitrarily chosen, $b_{l}{ }^{\mathrm{T}} \cdot b_{j}$ $=\min _{i \neq l, i=1,2, \ldots n-1} b_{l}^{\mathrm{T}} \cdot b_{i}$ where $j \in U=\{p, q, \quad r, \ldots$, $w\}(p \neq q \neq r \neq \ldots \neq w \neq l, 1 \leq p, q, r, \ldots, w \leq n-1)$. Thus, the $t_{h}, t_{m}$, $t_{n}, \ldots, t_{l}$ that correspond to $\bar{U}=\{h, m, n, \ldots, l\}$ construct a linear sub-tree of $T$, where $U \cap \bar{U}=\phi$ and $\mid U$ $\cup \bar{U} \mid=n-1$. Then for a certain $k \in \bar{U}$, if there is a certain $s \in U$ such that $b_{k}^{T} \cdot b_{s}=\min _{j \neq s, j=p, q, r, \ldots, w} b_{k}{ }^{T} \cdot b_{j}, t_{s}$ is a pendent branch of the linear tree $T$ that corresponds to $\mathbf{B}_{\mathbf{t}}$.

As is seen in Figure 5, $U=\{p, q, \mathrm{r}\}$ and $\bar{U}=\{h, m, n$, l). Let $k=h \in \bar{U}$ and $s=p \in U$. Since $b_{h}{ }^{T} \cdot b_{p}=1<b_{h}{ }^{T} \cdot b_{q}=2$ and $b_{h}{ }^{T} \cdot b_{p}=1<b_{h}{ }^{T} \cdot b_{r}=3, t_{p}$ is a pendent branch of the linear tree $T$.

\section{The Algorithm for the Construction of the Linear Tree}

We propose the following algorithm for the construction of the linear tree $T$. The thread of thinking is that one of the two pendent branches of $T$, e.g., $t_{p}$ is found first. The other pendent tree branch $t_{q}$ is found by using $t_{p}$ as a base. Then $t_{q}$ is taken off, the other pendent tree branch $t_{r}$ is found by using $t_{p}$ as a base again. Keep on with this procedure until the order of all the branches of $T$ is decided.

1) For a given fundamental loop matrix $\mathbf{B}_{\mathbf{f}}=\left[\mathbf{B}_{\mathbf{t}} \mathbf{1}\right]$, let $\mathbf{M}=\mathbf{B}_{\mathbf{t}}{ }^{\mathrm{T}} \mathbf{B}_{\mathbf{t}}$ where the entry $m_{i j}=b_{i}{ }^{\mathrm{T}} \cdot b_{j}$. Establish a ma-

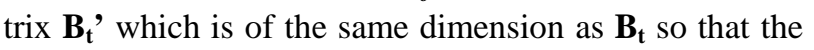
columns of $\mathbf{B}_{\mathbf{t}}$ after ordering can be put into $\mathbf{B}_{\mathbf{t}}$ '. Let the column index for $\mathbf{B}_{\mathbf{t}}{ }^{\prime}$ be $f$. Set $f=1$.

2) For row $i(1 \leq i \leq n-1)$ in $\mathbf{M}$ (Usually, let $i=1$ first to follow the row order in $\mathbf{M}$ ), if there is only one entry $m_{i p}$ in row $i$ that takes the minimum value, $t_{p}$ that corresponds to column $p$ is a pendent branch of the linear tree

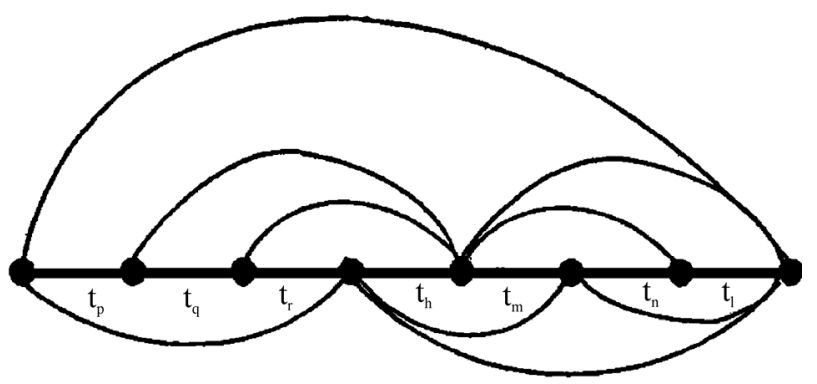

Figure 5. The explanation of Theorem 7. 
$T$ according to Theorem 3. Go to step (6). On the other hand, if there are multiple entries $m_{i p_{d}}, m_{i q_{d}}, m_{i r_{d}}, \ldots$, $m_{i w_{d}}$ in row $i$ that take the minimum value, there must exist a certain $s \in U_{d}=\left\{p_{d}, q_{d}, r_{d}, \ldots, w_{d}\right\}$ ( $d$ is the iteration index. Let $d=1$ first.) such that $t_{s}$ that corresponds to column $s$ is a pendent branch of the linear tree $T$ according to Theorem 4.

3) For row $k$ in $\mathbf{M}$ where $k \in \bar{U}_{d}=\{h, m, n, \ldots$, $l\}$ (Usually, let $k$ follow the row order in $\bar{U}_{d}$ ), if there is only one entry $m_{k s}$ in row $k$ that takes the minimum value where $s \in U_{d}, t_{s}$ that corresponds to column $s$ is a pendent branch of the linear tree $T$ according to Theorem 7 . Go to step (4). On the other hand, if there are multiple entries $m_{k p_{d}}, m_{k q_{d}}, m_{k r_{d}}, \ldots, m_{k w_{d}}$ in row $k$ that take the minimum value, there must exist a certain $s \in U_{d}=\left\{p_{d}\right.$, $\left.q_{d}, r_{d}, \ldots, w_{d}\right\}(d=d+1)$ such that $t_{s}$ that corresponds to column $s$ is a pendent branch of the linear tree $T$ according to Theorem 4. Repeat step (3) until $k$ takes all the elements in $\bar{U}_{d}$. At that time, if there are still multiple entries in row $k$ that take the minimum value, go to step (8).

4) If the last column of $\mathbf{B}_{\mathbf{t}}$ ' is not filled by a column from $\mathbf{B}_{\mathbf{t}}$ yet, set $p=s$. Go to step (6). Otherwise, judge the adjacency of $t_{k}, t_{s}$ and $t_{p}$ according to Theorem 2 .

5) If $t_{k}$ and $t_{p}$ are at the same side of $t_{s}$, i.e., the order is $t_{s}, \ldots, t_{k}, \ldots, t_{p}$, set $p=s$. Go to step (6). On the other hand, if $t_{k}$ and $t_{p}$ are at the different sides of $t_{s}$, i.e., the order is $t_{k}, \ldots, t_{s}, \ldots, t_{p}$, use $t_{k}$ as a pendent tree branch to find the order of the tree branches corresponding to $U_{d}$ and put them into the columns of $\mathbf{B}_{\mathbf{t}}$ ', i.e., column $f$ to column $f^{\prime}$ where $f^{\prime}=f+\left(\right.$ number of elements in $\left.U_{d}\right)-1$. Set all the entries in the columns of $\mathbf{M}$ corresponding to $U_{d}$ to be $\infty$. If the entries in $\mathbf{M}$ are all $\infty$, stop. If not, set $f=f+$ (number of elements in $U_{d}$ ). Go back to step (2).

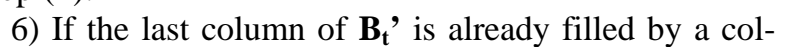
umn from $\mathbf{B}_{\mathbf{t}}$, i.e., a pendent tree branch at one end is already decided, go to step (b). Otherwise

a) Assume the pendent branch of $T$ is $t_{p}$. Put $t_{p}$ into the last column of $\mathbf{B}_{\mathbf{t}}$ '. Set $i=p$. Go to step (7).

b) Put column $p$ of $\mathbf{B}_{\mathbf{t}}$ into column $f$ of $\mathbf{B}_{\mathbf{t}}$ '. Set $f=f+1$.

7) If all the columns of $\mathbf{B}_{\mathbf{t}}$ have been put into $\mathbf{B}_{\mathbf{t}}$ ', the ordered columns of $\mathbf{B}_{\mathbf{t}}$ ' have already constitute a linear tree. Stop. Otherwise, set the entries in column $p$ of $\mathbf{M}$ to be $\infty$. Go back to step (2).

8) When there are only two entries in $U_{d}$, choose arbitrarily $s \in U_{d}$. $t_{s}$ is a pendent branch of $T$. Go to step (4). Otherwise, according to Theorem 5 and Theorem 6 , the linear sub-tree in graph $G_{1}$ that corresponds to $U_{d} *=U_{d}$ can be put into order separately. Thus, take the columns $\mathbf{B}_{\mathbf{t}}^{(1)}$ in $\mathbf{B}_{\mathbf{t}}$ that correspond to the elements in
$U_{d}$ to construct $\mathbf{B}_{\mathbf{t}}^{(1) \mathrm{T}} \mathbf{B}_{\mathbf{t}}^{(1)}=\mathbf{M}^{(1)}$. Repeat steps (2)-(8) for $\mathbf{M}^{(1)}$. If the number of elements in $U_{d}$ is not changed after one iteration, the order of the corresponding tree branches is arbitrary. Put the ordered columns of $\mathbf{B}_{\mathbf{t}}{ }^{(1)}$ into column $f$ to column $f$ ' where $f^{\prime}=f+\left(\right.$ number of elements in $\left.U_{d}\right)-1$. Set all the entries in the columns of $\mathbf{M}$ corresponding to $U_{d}$ to be $\infty$. If the entries in $\mathbf{M}$ are all $\infty$, stop. If not, set $f=f+$ (number of elements in $U_{d}$ ). Go back to step (2).

\section{An Example}

Given a fundamental loop matrix

$$
\mathrm{B}_{\mathrm{r}}=\left[\begin{array}{lllllllll}
1 & 2 & 3 & 4 & 5 & 6 & 7 & 8 & 9 \\
1 & 1 & 1 & 1 & 1 & 1 & 0 & 0 & 0 \\
1 & 0 & 0 & 0 & 1 & 0 & 1 & 0 & 0 \\
1 & 1 & 1 & 0 & 1 & 0 & 0 & 1 & 0 \\
0 & 0 & 1 & 1 & 1 & 0 & 0 & 0 & 1
\end{array}\right],
$$

we have

$$
\mathbf{B}_{\mathbf{t}}=\left[\begin{array}{lllll}
1 & 2 & 3 & 4 & 5 \\
1 & 1 & 1 & 1 & 1 \\
1 & 0 & 0 & 0 & 1 \\
1 & 1 & 1 & 0 & 1 \\
0 & 0 & 1 & 1 & 1
\end{array}\right] \text { and } \mathbf{B}_{\mathbf{t}}{ }^{\mathrm{T}}=\left[\begin{array}{llll}
1 & 1 & 1 & 0 \\
1 & 0 & 1 & 0 \\
1 & 0 & 1 & 1 \\
1 & 0 & 0 & 1 \\
1 & 1 & 1 & 1
\end{array}\right] .
$$

a) According to step (1),

$$
\mathbf{M}=\mathbf{B}_{\mathbf{t}}{ }^{\mathrm{T}} \mathbf{B}_{\mathbf{t}}={ }_{3}\left[\begin{array}{ccccc}
1 & 2 & 3 & 4 & 5 \\
2 & 2 & 2 & 1 & 3 \\
2 & 2 & 2 & 1 & 2 \\
2 & 2 & 3 & 2 & 3 \\
2 & 1 & 2 & 2 & 2 \\
1 & 2 & 3 & 2 & 4
\end{array}\right] .
$$

Also, establish a matrix $\mathbf{B}_{\mathbf{t}}{ }^{\text {' which }}$ is of the same dimension as $\mathbf{B}_{\mathbf{t}}$ so that the columns of $\mathbf{B}_{\mathbf{t}}$ after ordering can be put into $\mathbf{B}_{\mathbf{t}}$ '. Let the column index for $\mathbf{B}_{\mathbf{t}}{ }^{\prime}$ be $f$. Set $f=1$.

b) According to step (2), consider row 1 of $\mathbf{M}$. As $m_{14}$ is the only entry in row 1 that takes the minimum value, $t_{4}$ is one pendent branch of $T$. Go to step (6).

c) According to step (6)(a), put column 4 of $\mathbf{B}_{\mathbf{t}}$ into the last column of $\mathbf{B}_{\mathbf{t}}$, i.e.,

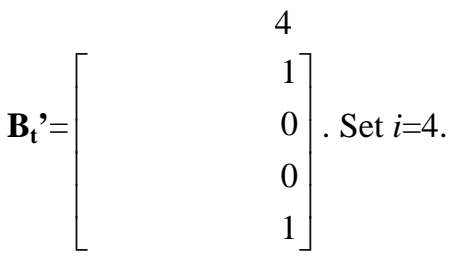


d) According to step (7), set all the entries in column 4 of $\mathbf{M}$ to be $\infty$, i.e.,

$$
\mathbf{M}=\frac{1}{2}\left[\begin{array}{lllll}
1 & 2 & 3 & 4 & 5 \\
3 & 2 & 2 & \infty & 3 \\
2 & 2 & 2 & \infty & 2 \\
2 & 2 & 3 & \infty & 3 \\
1 & 1 & 2 & \infty & 2 \\
3 & 2 & 3 & \infty & 4
\end{array}\right]
$$

e) According to step (2), consider row 4 of $\mathbf{M}$. As $m_{41}$ and $m_{42}$ are the entries in row 4 that take the minimum value, there must exist the other pendent branch of $T$ among $t_{1}$ and $t_{2}$ that correspond to $U_{1}=\{1,2\}$. Here, $\bar{U}_{1}=\{3,4,5\}$.

f) According to step (3), consider row 3 of $\mathbf{M}$. As $m_{31}$ and $m_{32}$ are the entries in row 3 that take the minimum value, there must exist the other pendent branch of $T$ among $t_{1}$ and $t_{2}$ that correspond to $U_{2}=\{1,2\}$. Here,

$\bar{U}_{2}=\{3,4,5\}$.

g) Repeat step (3). Consider row 5 of $\mathbf{M} . m_{52}$ is the only entry in row 5 that takes the minimum value.

h) According to step (4), as the last column of $\mathbf{B}_{\mathbf{t}}{ }^{\text {' }}$ is already filled by a column from $\mathbf{B}_{\mathbf{t}}$, judge the adjacency of $t_{5}, t_{2}$ and $t_{4}$. As $m_{42}<m_{45}$ in $m_{4}$, the order is $t_{2}, \ldots$, $t_{5}, \ldots, t_{4}$.

i) According to step (5), as $t_{5}$ and $t_{4}$ are at the same side of $t_{2}, t_{2}$ is the other pendent branch of $T$ that is based on $t_{4}$. Set $p=2$.

j) According to step (6)(b), put column 2 of $\mathbf{B}_{\mathbf{t}}$ into the first column of $\mathbf{B}_{\mathbf{t}}$, i.e., $\mathbf{B}_{\mathbf{t}}{ }^{\prime}=\left[\begin{array}{ll}2 & 4 \\ 0 & 1 \\ 1 & 0 \\ 0 & 0 \\ 0\end{array}\right]$. Set $f=1+1=2$.

k) According to step (7), set all the entries in column 2 of $\mathbf{M}$ to be $\infty$, i.e.,

$$
\mathbf{M}=\frac{1}{2}\left[\begin{array}{ccccc}
1 & 2 & 3 & 4 & 5 \\
3 & \infty & 2 & \infty & 3 \\
2 & \infty & 2 & \infty & 2 \\
2 & \infty & 3 & \infty & 3 \\
1 & \infty & 2 & \infty & 2 \\
3 & \infty & 3 & \infty & 4
\end{array}\right] .
$$

l) According to step (2), consider row 4 of $\mathbf{M}$. As $m_{41}$ is the only entry in row 4 that takes the minimum value, $t_{1}$ is the other pendent branch of $T$ that is based on $t_{4}$ with $t_{2}$ taken off.

m) According to step (6), put column 1 of $\mathbf{B}_{\mathbf{t}}$ into the second column of $\mathbf{B}_{\mathbf{t}}$, i.e., $\mathbf{B}_{\mathbf{t}}{ }^{\prime}=\left[\begin{array}{lll}2 & 1 & 4 \\ 1 & 1 & 1 \\ 0 & 1 & 0 \\ 1 & 1 & 0 \\ 0 & 0 & 1\end{array}\right]$. Set

$f=2+1=3$.

n) According to step (7), set all the entries in column 1 of $\mathbf{M}$ to be $\infty$, i.e.,

$$
\mathbf{M}=\frac{1}{2}\left[\begin{array}{ccccc}
1 & 2 & 3 & 4 & 5 \\
3 & \infty & 2 & \infty & 3 \\
5 & \infty & 2 & \infty & 2 \\
\infty & \infty & 3 & \infty & 3 \\
\infty & \infty & 2 & \infty & 2 \\
\infty & \infty & 3 & \infty & 4
\end{array}\right] .
$$

o) According to step (2), consider row 4 of M. As $m_{43}$ and $m_{45}$ are the entries in row 4 that take the minimum value, there must exist the other pendent branch of $T$ that is based on $t_{4}$ with $t_{1}$ and $t_{2}$ taken off among $t_{3}$ and $t_{5}$ that correspond to $U_{1}=\{3,5\}$. Here, $\bar{U}_{1}=\{1,2,4\}$.

p) According to step (3), consider row 1 of $\mathbf{M} . m_{13}$ is the only entry in row 1 that takes the minimum value.

q) According to step (4), as the last column of $\mathbf{B}_{\mathbf{t}}$ ' is already filled by a column from $\mathbf{B}_{\mathbf{t}}$, judge the adjacency of $t_{1}, t_{3}$ and $t_{4}$. As $m_{13}>m_{14}$ in $m_{1}$, the order is $t_{1}, \ldots, t_{3}, \ldots$, $t_{4}$.

r) According to step (5), as $t_{1}$ and $t_{4}$ are at the different sides of $t_{3}, t_{2}$ is used as the other pendent branch of $T$ to find the order of the tree branches corresponding to $U_{d}$. Put column 3 of $\mathbf{B}_{\mathbf{t}}$ into the fourth $\left(f^{\prime}=3+2-1=4\right)$ column of $\mathbf{B}_{\mathbf{t}}$, i.e.,

$$
\mathbf{B}_{\mathbf{t}}=\left[\begin{array}{llll}
2 & 1 & 3 & 4 \\
1 & 1 & 1 & 1 \\
0 & 1 & 0 & 0 \\
1 & 1 & 1 & 0 \\
0 & 0 & 1 & 1
\end{array}\right]
$$

Set $f=3+1=4$. Set all the entries in column 3 of $\mathbf{M}$ to be $\infty$, i.e.,

$$
\mathbf{M}=\frac{1}{1} \cdot\left[\begin{array}{ccccc}
1 & 2 & 3 & 4 & 5 \\
\infty & \infty & \infty & \infty & 3 \\
\infty & \infty & \infty & \infty & 2 \\
\infty & \infty & \infty & \infty & 3 \\
\infty & \infty & \infty & \infty & 2 \\
\infty & \infty & \infty & \infty & 4
\end{array}\right] .
$$

s) According to step (2), consider row 4 of $\mathbf{M}$. As $m_{45}$ is the only entry in row 4 that takes the minimum value, $t_{5}$ is the other pendent branch of $T$ that is based on $t_{1}$ with 
$t_{3}$ taken off.

t) According to step (6), put column 5 of $\mathbf{B}_{\mathbf{t}}$ into the third column of $\mathbf{B}_{\mathbf{t}}$, i.e., $\mathbf{B}_{\mathbf{t}}{ }^{\prime}=\left[\begin{array}{lllll}2 & 1 & 5 & 3 & 4 \\ 1 & 1 & 1 & 1 & 1 \\ 0 & 1 & 1 & 0 & 0 \\ 1 & 1 & 1 & 1 & 0 \\ 0 & 0 & 1 & 1 & 1\end{array}\right]$. Set $f=4+1=5$.

u) According to step (7), stop.

As a summary, we have the following table to achieve the order of the columns in $\mathbf{B}_{\mathbf{t}}$ '.

\begin{tabular}{|c|c|c|c|c|c|}
\hline Colu. in $\mathbf{B}_{\mathbf{t}}{ }^{\prime}$ & 5 & 1 & 2 & 4 & 3 \\
\hline Colu. in $\mathbf{B}_{\mathbf{t}}$ & 4 & 2 & 1 & 3 & 5 \\
\hline Steps & (b),(c),(d) & $\begin{array}{c}\text { (e),(f),(g), } \\
\text { (h),(i),(j), } \\
\text { (k) }\end{array}$ & (l),(m),(n) & $\begin{array}{c}\text { (o),(p), } \\
\text { (q),(r) }\end{array}$ & (s),(t),(u) \\
\hline $\begin{array}{c}\text { According to } \\
\text { Algorithm } \\
\text { Steps }\end{array}$ & (2),(6), (7) & $\begin{array}{c}\text { (2),(3),(3),,(5),, } \\
\text { (7) }\end{array}$ & (2),(6),(7) & $\begin{array}{c}\text { (2),(3), } \\
\text { (4),(5) }\end{array}$ & (2),(6),(7) \\
\hline
\end{tabular}

The column order [2, 1, 5, 3, 4] of $\mathbf{B}_{\mathbf{t}}{ }^{\prime}$ is thus the order of the branches of the linear tree as shown by the bold segments in Figure 6. The graph that corresponds to $\mathbf{B}_{\mathbf{f}}$ can then be obtained by adding the links $6,7,8$ and 9.

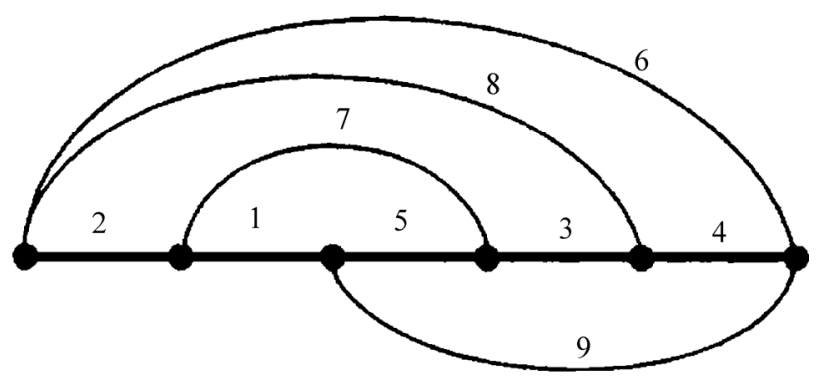

Figure 6. The graph that corresponds to $B_{f}$.

\section{Conclusions}

This paper presents an algorithm for the realization of a linear tree based on the judgment of the pendent properties of the tree branches and the determination of their order one by one. The graph that corresponds to $\mathbf{B}_{\mathrm{f}}$ is eventually constructed by adding links to the obtained linear tree. As an arbitrary tree contains a linear tree, the linear tree can then be realized first to realize a general tree. This will be discussed in another paper of ours.

The main contribution of this paper lies in the proposition of a new approach to the realization of a linear tree. Experiments validate the effectiveness of the proposed approach. This lays a foundation to the realization of a general tree and therefore a random graph from a given matrix.

\section{References}

[1] L. Q. Lei and B. Q. Dai, “A convenient method for formulation of a node incidence matrix from a basic cutset matrix,” (In Chinese), Journal of Jiangxi Polytechnic University, Vol. 14, No. 3, September 1992.

[2] W. Mayeda, “Graph theory,” John Wiley, New York, 1972.

[3] K. P. Rajappan, “On Okada’s method for realizing cutset matrices,” Journal of Combinational Theory, Vol. 10, pp. 135-142, 1971.

[4] M. N. S. Swamy and K. Thulasiraman, "Graph, network and algorithms,” John Wiley, New York, 1981.

[5] L. Zhu, "An expression for the relationship between the incidence Matrix A of Graph G and the basic loop Matrix $\mathrm{B}_{\mathrm{f}}$," (In Chinese), Teaching and Scientific Technology, No. 1, pp. 72-75, March 1996.

[6] R. B. Ash and W. H. Kim, "On realizability of a circuit matrix,” IRE Transactions on Circuit Theory, Vol. CT-6, pp. 219-223, June 1959.

[7] S. R. Parker and H. J. Lohse, "A direct procedure for the synthesis of network graphs from a given fundamental loop or cutset matrix," IEEE Transactions on Circuit Theory, Vol. CT-16, pp. 221-223, May 1969. 